Please do not remove this page

RMIT

UNIVERSITY

\title{
Black phosphorus: ambient degradation and strategies for protection
}

Kuriakose, Sruthi; Ahmed, Taimur; Balendhran, Shiva; Bansal, Vipul; Sriram, Sharath; Bhaskaran, Madhu; Walia, Sumeet

https://researchrepository.rmit.edu.au/esploro/outputs/9921862924501341/filesAndLinks?institution=61RMIT_INST\&index=null

Kuriakose, S., Ahmed, T., Balendhran, S., Bansal, V., Sriram, S., Bhaskaran, M., \& Walia, S. (2018). Black phosphorus: ambient degradation and strategies for protection. 2D Materials, 5, 1-10.

https://doi.org/10.1088/2053-1583/aab810

Document Version: Accepted Manuscript

Published Version: https://doi.org/10.1088/2053-1583/aab810

Repository homepage: https://researchrepository.rmit.edu.au

(c) 2018 IOP Publishing Ltd

Downloaded On 2023/04/26 12:31:58 +1000

Please do not remove this page 
Thank you for downloading this document from the RMIT Research Repository.

The RMIT Research Repository is an open access database showcasing the research outputs of RMIT University researchers.

RMIT Research Repository: http://researchbank.rmit.edu.au/

\section{Citation:}

Kuriakose, S, Ahmed, T, Balendhran, S, Bansal, V, Sriram, S, Bhaskaran, M and Walia, S 2018, 'Black phosphorus: ambient degradation and strategies for protection', 2D Materials, vol. 5, no. 3, 032001, pp. 1-10.

See this record in the RMIT Research Repository at:

https://researchbank.rmit.edu.au/view/rmit:47642

Version: Accepted Manuscript

Copyright Statement:

(C) CC BY-NC-ND 3.0 licence

https://creativecommons.org/licenses/by-nc-nd/3.0/

Link to Published Version:

https://dx.doi.org/10.1088/2053-1583/aab810 


\section{Black Phosphorus: Ambient degradation and strategies for protection}

Sruthi Kuriakose, Taimur Ahmed, Sivacarendran Balendhran, Vipul Bansal, Sharath Sriram, Madhu Bhaskaran and Sumeet Walia*

Ms. S. Kuriakose, Mr. T. Ahmed, Dr. S. Balendhran, Assoc. Prof. S. Sriram, Assoc. Prof. M. Bhaskaran, Dr. S. Walia

Functional Materials and Microsystems Research Group and Micro Nano Research Facility, RMIT University, Melbourne VIC 3001, Australia

*E-mail: sumeet.walia@ rmit.edu.au

Prof. V. Bansal

Ian Potter NanoBioSensing Facility and NanoBiotechnology Research Laboratory, School of Science, RMIT University, Melbourne VIC 3001, Australia

Keywords: black phosphorus, phosphorene, degradation, protection, review

Elemental two-dimensional black phosphorus (BP) is a highly anisotropic versatile material capable of exhibiting wide ranging electronic characteristics ranging from semi-metallic to semiconducting. Its thickness dependent tunable energy gap makes it an exciting prospect for deployment in a variety of applications. The main hurdle limiting diverse applications incorporating BP is its ambient instability. BP degrades rapidly under room conditions, affecting its structure and properties. In this report, we cover the recent progress that has occurred towards protecting BP from ambient degradation. We review the major developments in effectively countering the problem and compare their relative degrees of success. This is provided in the context of the mechanisms governing the atmospheric instability of this material. A targeted focus is kept on the various causes of degradation of BP in atmospheric conditions and the protection strategies that have been implemented so far.

\section{Introduction}

Two-dimensional (2D) materials have attracted unprecedented attention over the past decade for their striking properties that are not normally present in their bulk form. Recently, 2D 
black phosphorus (BP) and its monolayer form (phosphorene) have emerged as an attractive elemental analogue to graphene. It possesses a thickness-dependent band gap that ranges between $0.3 \mathrm{eV}$ to $2.0 \mathrm{eV}{ }^{[1]}$ and carrier mobilities of the order of $1000 \mathrm{~cm}^{2} \mathrm{~V}^{-1} \mathrm{~S}^{-1}$. ${ }^{[2]}$ The relatively weak van der Waal forces holding the interlayers of the material and the strong inplane bonding forces enables seamless exfoliation of BP similar to that of graphene and other transition metal dichalcogenides (TMDs). ${ }^{[3,4]} \mathrm{BP}$ has been shown to possess fascinating properties that can be harnessed for a large variety of applications such as energy storage devices $^{[2]}$, field effect transistors ${ }^{[5,6]}$, thermoelectrics ${ }^{[5]}$, broad spectrum photodetectors and a variety of sensors $^{[5,7-9]}$. When compared to other well-known $2 \mathrm{D}$ materials (such as graphene and TMDs $)^{[4,10]}$, BP presents significant advantages such as an intrinsically direct band gap regardless of thickness and highly anisotropic electronic, optical ${ }^{[11,12]}$ and thermal transport $^{[13,14]}$ that allows engineering its properties based on the crystal orientation. ${ }^{[1,15,16]}$ However, a major hurdle in the deployment of this material in practical applications, is its environmental instability. This has led to a parallel body of studies that have investigated the underlying mechanism of BP degradation and techniques to prevent the ambient deterioration of BP.

In this article, we identify a cohort of factors and corresponding mechanisms that result in the degradation of BP. This perspective is not aimed to be a comprehensive review of the large volume of literature on the synthesis and applications of BP which are already covered in recent articles. ${ }^{[1,3,5,16-20]}$ Rather, this is a focussed overview of the proposed degradation mechanisms and the strategies to prevent material deterioration that have been explored tilldate. We also offer potential applications of BP once stability concerns are effectively addressed.

\section{Degradation of Black Phosphorus}


Despite the tremendous potential of BP for a range of applications outlined earlier, progress towards practical implementation of BP-based devices has stalled due to its rapid ambient degradation. This has turned the recent focus from exploring new applications to examining the fundamental causes of degradation and preventive/curative pathways to preserve the material. If a reliable solution to this problem is not established, there is general consensus that this rather unique material cannot be translated from the academic laboratories to realworld applications. If it does happen however, there is an array of opportunities that span across disciplines. In an ambient environment, the major factors that may potentially lead to BP degradation are light, oxygen, humidity and temperature. In this section, we will discuss the contribution of these key factors towards BP degradation.

\subsection{Light and Oxygen}

The initial studies that investigated the mechanism of BP degradation pointed to humidity and oxygen as the main contributor and a negligible effect of temperature. ${ }^{[21-26]}$ Parallel investigations also indicated the dominant role played by photo-oxidation in BP degradation. ${ }^{[27]}$ Collectively, these reports implied that the exposure of BP to ambient light results in the formation of reactive oxygen species (ROS) on the surface that eventually degrade the material. ${ }^{[28]}$ In many ways, this is analogous to the photosystem II chemistry in plants wherein oxidative species toxic to organisms are formed mainly due to the interaction of light with environmental oxygen. ${ }^{[29]}$ In such a process, there has to be a combination of factors at play which are indeed the case for BP degradation. ${ }^{[30]}$ Therefore, a controlled study looked at the isolated influence of light, oxygen and humidity. The influence of light on the formation of damaging ROS on the BP surface has to be studied in conjunction with its interplay with the environmental oxygen. The formation of these ROS is a result of lightinduced oxidation of the BP surface. ${ }^{[30]}$ Studies have revealed that oxygen can readily be chemisorbed on to the surface of BP resulting in an exoenergetic reaction introducing neutral defects at the atomic scale. ${ }^{[31]}$ These defects have been examined both theoretically and 
experimentally. It has been shown that these defects increase the lattice spacing promoting further oxidation in the pre-existing oxygen defects and eventually forming oxide compounds. $[26,31-33]$

This process is also affected by the crystal thickness. For monolayers, essentially all atoms are on the surface or at the interfaces; hence, the number of surface and interfacial defects per atom is greatly enhanced compared to thicker crystals. Therefore in thinner BP, it is typical to observe that the degradation initiates from the edges and progresses towards the middle, whereas thicker BP is predominantly prone to surface degradation. ${ }^{[34]}$ This results in a higher degradation rate for thinner BP layers. ${ }^{[35]}$ Another factor to consider is that the band gap of BP shifts towards higher energies as the thickness reduces, bringing it closer to $\mathrm{O}_{2}$ acceptor states which consequently increases the rate of charge transfer and hence, resulting in faster oxidation. As a result, typically, thinner BP layers are more readily oxidised compared to the thicker layers.

As such, the light induced oxidation comprises of three critical steps as shown in Figure 1(a) ${ }^{[27,28]}$ :

1) The light when incident on BP in ambient conditions, produces ROS:

$$
O_{2}(g)+h v \stackrel{\text { BP }}{\rightarrow} O_{2}^{-}+h^{+}
$$

where, BP denotes black phosphorus and $h^{+}$denotes the majority carrier holes.

2) The photogenerated $O_{2}^{-}$forms two $\mathrm{P}-\mathrm{O}$ bonds at the $\mathrm{BP}$ surface, resulting in a native surface oxide.

$$
O_{2}^{-}+P+h^{+} \rightarrow P_{x} O_{y}
$$

3) The formed oxide species interact with humidity and result in the sequential removal of $\mathrm{O}_{2}$ and $\mathrm{P}$ atoms from the $\mathrm{P}-\mathrm{O}$ bonds, leading to the disintegration of the surface of the $\mathrm{BP}$ layer opening up the layer below it for further degradation.

$$
P_{x} O_{y}+H_{2} O \stackrel{h+}{\rightarrow} P_{x}^{+}+O_{y}^{-}
$$


Therefore it is evident that BP degradation is triggered due to surface photo-oxidation, without which the reaction will not be able to proceed. In order to devise a comprehensive strategy for protecting BP under ambient conditions, it is also important to narrow down the band of wavelength that triggers the photo oxidation. Although theoretical studies have been performed to determine the oxidation energies in the BP band structure to predict the wavelengths of maximum damage, ${ }^{[36]}$ it is only recently that it was confirmed experimentally. ${ }^{[30]}$ Based on a time based topographic analysis, it can be seen that the UV bandwidth of the spectrum causes maximum degradation (Figure 2). In fact, wavelengths higher than those corresponding to the green light did not show any surface or electrical property degradation as reported in the study. Furthermore, it is reported that if the BP is isolated from UV light, it can be preserved from degradation for longer periods. ${ }^{[30]}$

\subsection{Humidity}

The rate of $\mathrm{BP}$ degradation in the presence of only water or moisture has been shown to be negligible compared to that in air or light. ${ }^{[32]}$ However, the co-absorption of water and oxygen on BP surface has been observed to speed up the deterioration of $\mathrm{BP}$ even in a dark environment. Pristine BP is hydrophobic, but becomes hydrophilic once it is oxidises. $^{[28,30,34,35]}$ The water adsorption energy increases as the BP surface becomes hydrophilic due to oxidation. ${ }^{[26]}$ A study has examined the role of oxygen and humidity in detail. In this study, pristine BP was kept in deionized water which did not suffer any degradation. ${ }^{[26]}$ Upon subsequent exposure to ambient air, the material completely degraded leaving behind traces of the BP (sequence shown in Figure 3(a-d). Thereafter, a comparison was carried out for BP immersed in deaerated water and oxygen rich water. While the BP in deaerated water did not show discernible signs of degradation, samples stored in oxygen rich water completely degraded within 2 days (Figure 3(e-h). This highlights the key role of oxygen in the degradation of BP. ${ }^{[26]}$ When water reacts with the oxidised BP, phosphoric acid (shown in Figure 1(b)) species $\left(\mathrm{H}_{x} \mathrm{PO}_{y}\right)^{[22,26,32]}$ are formed on the surface making the 
underlying layer prone to further oxidation. The detrimental effects of humidity are further demonstrated using a FET based study in different humidity conditions (Figure 3(i-j). ${ }^{[37]}$ The degradation is slower in deaerated water as they are devoid of oxygen. ${ }^{[26]}$ As the pristine BP surface is hydrophobic, water only gets weakly physisorbed on to its surface. The interaction of BP with water is strongly affected by the surface oxidation. ${ }^{[21,26]}$ During this interaction, there is a formation of a H-bond as explained in Equation (3) ${ }^{[28]}$ with the oxygen on the oxidised surface. A recent study also postulates that deionized water can be utilised to simply wash off the oxide layer from the top surface of BP. ${ }^{[38]}$ However, the effectiveness of this process is dampened by the observed deterioration in electrical characteristics. ${ }^{[24]}$

\section{Protection Strategies}

So far, we have focussed on the mechanisms of BP degradation. As it is now reasonably wellestablished that photo-oxidation expedited by humidity deteriorates this otherwise exotic material, we now look at various protection strategies that have been developed till-date. There are predominantly two routes that have been explored for BP stabilisation, namely, physical and chemical processes. The physical routes involve the incorporation of protection layers which isolates BP from the environment, whereas the chemical route rely on surface treatment of BP in an effort to transform it from environmentally active to being inert. A brief summary of the protection strategies and outcomes is presented in Table $\mathbf{1 .}$

\subsection{Physical Routes: Passivation layers}

Application of a range of passivation layers have been studied for the physical protection of BP. These include layers of dielectric materials listed in the Table 1 along with the methods employed to deposit them. The use of ionophore coating as passivation layer showing stability up to a month has also been reported. ${ }^{[7]}$

However, the most commonly used and effective passivation layer has been $\mathrm{Al}_{2} \mathrm{O}_{3}$ which limits the interaction of air with the BP surface, thereby minimising degradation (maximum 
stability period demonstrated to be 17 months). ${ }^{[39]}$ The $\mathrm{Al}_{2} \mathrm{O}_{3}$ passivation layers (shown in Figure 4f) are usually deposited on the top of BP by using the atomic layer deposition (ALD) and plasma enhanced ALD (PEALD) techniques in repeated cycles with $\mathrm{O}_{2}$ or $\mathrm{H}_{2} \mathrm{O}$ precursors. The long term stability provided by $\mathrm{Al}_{2} \mathrm{O}_{3}$ may be linked back to a previous study that reported ${ }^{[30]} \mathrm{UV}$ light to cause maximum photo-oxidation. It is known that $\mathrm{Al}_{2} \mathrm{O}_{3}$ attenuates UV light and coupled with its low oxygen diffusion constant inhibits the photo-oxidation process. ${ }^{[40-42]}$ However, further studies may be needed to verify this aspect. The fact remains that from the time of exfoliation/deposition of BP till the time it is encapsulated, oxide species are inevitably formed on the surface and it is impractical to assume otherwise. This is one of the reasons researchers try to process/characterize $\mathrm{BP}$ in an inert environment prior to the encapsulation. Now this is counterintuitive in more ways than one. Firstly, it demands the use of an inert environment albeit temporarily and secondly, an encapsulation layer limits the room for material engineering by preventing physical access to BP. This encapsulating layer, in particular, may drastically limit the application of BP for real-world applications, for instance, through adversely influencing the mechanical properties of flexible/wearable devices, and/or affecting the overall optical transparency of such devices, limiting their optoelectronic applications. ${ }^{[43-45]}$ Another practical problem is that physical or chemical deposition techniques (such as ALD, CVD and MOCVD) ${ }^{[46-50]}$ cannot be reliably used for the passivation of thin [a monolayer or even few-layer $(<5$ layers $)$ ] BP samples, because such thin layers are too unstable ${ }^{[30,35,47]}$ and are rapidly oxidized during the deposition processes. This can be overcome by capping with materials such as boron nitride but it is a complex process and has an extremely low throughput and yield. ${ }^{[49,51,52]}$

The relative success of implementing passivation layers has motivated studies into employing protective layers using other 2D materials. Furthermore, it can be expected that with an informed choice, key properties such as carrier mobilities can actually be enhanced. These are briefly discussed next. 


\subsection{Physical Routes: Capping using other 2D materials}

Hybrids comprising of BP stacked under other 2D materials have been investigated as one possible route towards ambient protection of BP. The underlying rationale is similar to capping wherein a physical barrier is introduced between BP and the environment. Although coverage with standalone or a combination of layers (from Table 1) of hexagonal Boron Nitride $(\mathrm{hBN})^{[47,53-55]}$, graphene ${ }^{[50]}$, and molybdenum sulphide $\left(\mathrm{MoS}_{2}\right)^{[46]}$ have been successful in improving stability without compromising electrical properties of BP (shown in Figure 4(a),(b) and (d)), it is not a viable method as it alters a range of optical and charge transfer characteristics. This limits optoelectronic applications ${ }^{[20]}$ for instance, as other $2 \mathrm{D}$ materials depending on their band energy alignments block certain wavelengths which BP inherently is capable of responding. In particular the wavelengths used in telecommunications ranging 0.8 to $1.7 \mu \mathrm{m} .{ }^{[56,57]} \mathrm{BP}$ is unique in the fact that it is highly anisotropic both electrically ${ }^{[12,19]}$ and thermally ${ }^{[13,14]}$. Moreover, it possesses a direct band gap regardless of thickness. ${ }^{[18]}$ This gives it unprecedented versatility in terms of implementing it for an almost uncounted array of applications. Most 2D materials cannot boast of the same characteristics and their incorporation can limit the breadth of applications even though they may enhance some selected properties such as carrier mobilities. ${ }^{[58]}$ Another challenge is to accurately place the encapsulating 2D material so as to cover the BP fully. The most widely implemented techniques of exfoliation carry a disadvantage of being unpredictable in terms of the location where the crystals would be deposited. This can be minimised by precisely controlling the alignment, but the uneven size yield for most 2D materials does not guarantee full coverage of the underlying BP which can still make it prone to degradation even though the rate of deterioration might be relatively slower compared to unprotected $\mathrm{BP} .{ }^{[47,59]}$

\subsection{Chemical Routes: Surface modification by organic compounds}

In order to overcome the limitations imposed by encapsulating the BP layers with an aim to isolate it from the ambient environment, another promising pathway has emerged recently. 
This typically involves the surface treatment of BP with chemical species of various types. The approaches that have shown success so far have been surface functionalization of the BP theoretically with metoxybenzene (MB), nitrobenzene (NB), and poly(phenylenevinylene $)^{[59]}$, and experimental tests of surface treatment (from Table 1) with N-cyclohexyl-2-pyrrolidone $(\mathrm{CHP})^{[25]}$, aryl diazonium ${ }^{[60]}$, octadecyltrichlorosilane $\mathrm{e}^{[61]}$ and ionic liquids like 1-butyl-3-

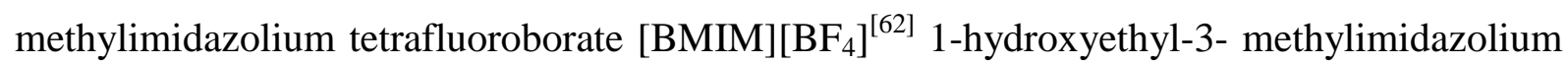
trifluoromethansulfonate ([HOEMIM]- $[\mathrm{TfO}]^{[63]}$ and 1-ethyl-3-methylimidazolium tetrafluoroborate $[\mathrm{EMIM}]\left[\mathrm{BF}_{4}\right]$ in acetonitrile $(\mathrm{MeCN}) .{ }^{[64]}$ In a number of these strategies, certain chemical species were found to influence the electronic characteristics of BP through surface charge doping, while remained inert. In cases, where chemical species induce doping states in BP, this could either be advantageous or detrimental to the overall (opto)electronic properties of the protected materials, and as-such in-depth investigations need to be undertaken to sequentially screen for the most appropriate chemical species suitable for BP protection. Among various studies, the use of $[\mathrm{BMIM}]\left[\mathrm{BF}_{4}\right]$ ionic liquid is particularly interesting, as this study employed a new strategy to directly target against the mechanism of BP photooxidative degradation. ${ }^{[62]}$ In this case, the $[\mathrm{BMIM}]\left[\mathrm{BF}_{4}\right]$ ionic liquid was chosen as an antioxidant molecule that could capture the ROS species generated on the BP surface, and therefore protect it from photodegradation while retaining its electronic characteristics. The treatment was limited to successfully binding antioxidative molecules to the surface, which was also confirmed by the density function theory (DFT) calculations shown in Figure 5(a), (b) and (d). These calculations reveal the binding energies of various molecules to different $\mathrm{BP}$ planes and a direct correlation between the binding energies and the effectiveness in stabilising BP.

This antioxidative pathway of surface treatment eliminates the need for isolating the BP from the atmosphere. Therefore, even if ROS have formed prior to treatment, they can be 
potentially removed by these antioxidants, which are naturally capable of scavenging the ROS ${ }^{[62,65]}$ that cause degradation. It has also been shown that the attachment of these species do not alter any of the fundamental properties of $\mathrm{BP}^{[25]}$ and the material remains fully accessible for vast range of applications including UV and humidity sensors ${ }^{[66,67]}$, which is otherwise not possible in the case of encapsulated BP. Furthermore, as the surface treatment covers a large area, it is scalable and removes the complexity faced in $2 \mathrm{D}$ encapsulation techniques that are impractical for large scale production. As a matter of fact, many other molecules that have been observed to provide photo stability to BP (Table 1) seem to have varying levels of antioxidant properties, which makes one wonder whether explicit use of antioxidants may offer a promising avenue for the ambient protection of BP. Taking a cue from this observation, liquid exfoliation methods have incorporated such compounds as solvents, wherein the exfoliated BP layers are readily functionalised to quench ROS.${ }^{[68]}$ However, as the surface structure of phosphorene is puckered in nature, a uniform chemical functionalisation may not necessarily be easy to achieve. This can potentially result in exposed/untreated areas at the atomic scale that can eventually act as oxidative sites to initiate the process of degradation even though lifetimes of these chemically-functionalised materials may still be significantly higher than untreated surfaces. This problem can be overcome by carefully choosing antioxidative molecules with matching chemical potentials that can lead to stronger binding at the surface. ${ }^{[69,70]}$

\section{Future Outlook}

Layered elemental analogues of graphene have been known for a long time; however the exploration of their properties in their two-dimensional forms is a relatively recent occurrence, with important implications for nanoelectronics and optoelectronics in particular. The band gaps of many of these elemental semiconductors make them highly attractive channel 
materials for transistors. Phosphorene (one of the analogues) possesses a bandgap in the "Goldilocks" zone for most electronic and optoelectronic applications. Moreover, the bandgap is thickness dependent and hence tunable. Applications in field effect transistors with high switching ratios and chemical/gas sensors have already been demonstrated with phosphorene as the functional layer. The lone but crippling problem is its nearly spontaneous oxidation in ambient conditions. While a lot of studies are now directed towards addressing this issue for BP, other elemental analogues of the 'enes' family, including boronene/borophenes ${ }^{[71]}$, silicene $^{[72]}$, germanene $e^{[73,74]}$, stanene $e^{[75]}$, plumbene $e^{[76]}$, arsenene ${ }^{[77]}$, antimonene ${ }^{[78,79]}$ and bismuthene ${ }^{[80,81]}$ remain on the fringes of research.

In terms of BP, its limited stability in the presence of light and oxygen and the eventual formation of phosphoric acid species in the presence of moisture is proving to be a technological challenge. ${ }^{[2,24,38]}$ However, once surmounted it is likely that focus will shift towards exploring large-scale fabrication which comes with its own set of challenges. A significant aspect of this review has focussed on critically outlining the pros and cons of two most common approaches of BP protection, viz. physical and chemical passivation strategies. Based on the cons of using a blanket encapsulation approach using an inorganic passivating layer discussed in the paper, we believe that this process is currently not ideal as a universal strategy for large-scale manufacturing of devices and products. However, considering the well-established success of physical deposition processes in industrial environments, such protection strategies may find niche prospects in devices targeted for specific applications, e.g. through employing an appropriate inorganic passivating layer. In comparison, moving forward, surface treatment options could offer a promising universal and versatile alternative strategy applicable for a wider range of devices and materials; but those methods remain to be fully matured, and require further developments to achieve perfection and fully establish themselves. In particular, a wide range of potential chemical protectants need to be actively hunted to screen the most promising candidate that offers ideal long-term protection of BP 
without influencing its electronic properties. This search for new chemical protectants may actually reveal interesting molecules that can further modulate and enrich the existing diverse portfolio of opto-electronic properties offered by BP. Further, in studying the physics and chemistry of BP and other elemental analogues, researchers should be able to draw upon the large body of work on graphene, transition metal oxides and chalcogenides, their intercalation chemistry, materials processing and device fabrication techniques. This is important, as 2D "enes" have many distinctive properties that are not seen in other material families, and as research progresses further, there are sure to be unexpected and exciting discoveries. In the next few years, progress in this field will require advances in scalable and controllable sample preparation to make large amounts of atomically thin and uniform layers, either in solutions or on substrates. For solution-phase fabrication, the challenge has always been to optimise the thickness and lateral dimensions as both these parameters counter each other. New methods that can efficiently and safely produce these materials in a defect-free manner and in large volumes need have to be further explored. For solid-state synthesis, crystal growth techniques for large-area growth of high-quality crystals with control over the number of layers needs to be achieved. Access to high-quality 2D materials will enable researchers to better understand the physical and chemical properties of these materials, as well as create a pathway for a wide variety of applications. As such, despite the challenges, elemental analogues offer an exciting and relatively niche area of exploration that has the potential to impart significant benefits to the nanotechnology community.

\section{Acknowledgements}

The authors acknowledge support from the Australian Research Council (ARC) for personnel and project support via DE150100909 (S.B.), FT140101285 (V.B.), DP170103477 (V.B.), DE160100023 (M.B.), DP140100170 (M.B.) 


\section{References}

[1] K. Cho, J. Yang, and Y. Lu," J. Mater. Res.,2017. 32, 2839.

[2] R. Gusmao, Z. Sofer, and M. Pumera," Angew. Chem-Ger Edit,2017. 129, 8164.

[3] A. J. Mannix, B. Kiraly, M. C. Hersam, and N. P. Guisinger," Nat. Rev. Chem.,2017. 1, 0014.

[4] A. R. Rezk, S. Walia, R. Ramanathan, H. Nili, J. Z. Ou, V. Bansal, J. R. Friend, M. Bhaskaran, L. Y. Yeo, and S. Sriram," Adv. Opt. Mater,2015. 3, 888.

[5] V. Eswaraiah, Q. Zeng, Y. Long, and Z. Liu," Small,2016. 12, 3480.

[6] R. A. Doganov, E. C. O’Farrell, S. P. Koenig, Y. Yeo, A. Ziletti, A. Carvalho, D. K. Campbell, D. F. Coker, K. Watanabe, and T. Taniguchi," Nat. Commun.,2015. 6, 7647.

[7] P. Li, D. Zhang, J. Liu, H. Chang, Y. e. Sun, and N. Yin," ACS Appl. Mater. Inter.,2015. 7, 24396.

[8] L. Kou, T. Frauenheim, and C. Chen," J. Phys. Chem. Lett.,2014. 5, 2675.

[9] S. Cui, H. Pu, S. A. Wells, Z. Wen, S. Mao, J. Chang, M. C. Hersam, and J. Chen," Nat. Commun.,2015. 6, 8632.

[10] S. Walia, S. Balendhran, Y. Wang, R. Ab Kadir, A. Sabirin Zoolfakar, P. Atkin, J. Zhen Ou, S. Sriram, K. Kalantar-zadeh, and M. Bhaskaran," Appl. Phys. Lett,2013. 103, 232105.

[11] J. Wu, N. Mao, L. Xie, H. Xu, and J. Zhang," Angew. Chem-Ger Edit, 2015. 127, 2396.

[12] J. Qiao, X. Kong, Z.-X. Hu, F. Yang, and W. Ji," Nat. Commun.,2014. 5, 4475.

[13] Z. Luo, J. Maassen, Y. Deng, Y. Du, R. P. Garrelts, M. S. Lundstrom, D. Y. Peide, and X. Xu," Nat. Commun.,2015. 6, 8572

[14] A. Łapińska, A. Taube, J. Judek, and M. Zdrojek," J. Phys. Chem. C,2016. 120, 5265.

[15] J.-S. Kim, Y. Liu, W. Zhu, S. Kim, D. Wu, L. Tao, A. Dodabalapur, K. Lai, and D. Akinwande," Sci. Rep-UK,2015. 5, 8989.

[16] M. Akhtar, G. Anderson, R. Zhao, A. Alruqi, J. E. Mroczkowska, G. Sumanasekera, and J. B. Jasinski," npj 2D Mater. Appl.,2017. 1, 5.

[17] G. Wang, R. Pandey, and S. P. Karna," Nanoscale, 2015. 7, 524.

[18] S. C. Dhanabalan, J. S. Ponraj, Z. Guo, S. Li, Q. Bao, and H. Zhang," Adv. Sci.,2017. 4, 1600305. 
[19] H. Liu, A. T. Neal, Z. Zhu, Z. Luo, X. Xu, D. Tománek, and D. Y. Peide," 2014.

[20] D. Y. Qiu, F. H. da Jornada, and S. G. Louie," Nano Lett.,2017. 17, 4706.

[21] S. Walia, Y. Sabri, T. Ahmed, M. R. Field, R. Ramanathan, A. Arash, S. K. Bhargava, S. Sriram, M. Bhaskaran, and V. Bansal," 2D Mater.,2016. 4, 015025.

[22] S.-L. Yau, T. P. Moffat, A. J. Bard, Z. Zhang, and M. M. Lerner," Chem. Phys. Lett,1992. 198, 383.

[23] J. D. Wood, S. A. Wells, D. Jariwala, K.-S. Chen, E. Cho, V. K. Sangwan, X. Liu, L. J. Lauhon, T. J. Marks, and M. C. Hersam," Nano Lett.,2014. 14, 6964.

[24] A. Castellanos-Gomez, L. Vicarelli, E. Prada, J. O. Island, K. Narasimha-Acharya, S. I. Blanter, D. J. Groenendijk, M. Buscema, G. A. Steele, and J. Alvarez," 2D Mater.,2014. 1, 025001.

[25] D. Hanlon, C. Backes, E. Doherty, C. S. Cucinotta, N. C. Berner, C. Boland, K. Lee, A. Harvey, P. Lynch, and Z. Gholamvand," Nat. Commun.,2015. 6, 8563.

[26] Y. Huang, J. Qiao, K. He, S. Bliznakov, E. Sutter, X. Chen, D. Luo, F. Meng, D. Su, and J. Decker," Chem. Mater.,2016. 28, 8330.

[27] A. Favron, E. Gaufrès, F. Fossard, A.-L. Phaneuf-L'Heureux, N. Y. Tang, P. L. Lévesque, A. Loiseau, R. Leonelli, S. Francoeur, and R. Martel," Nat. Mater.,2015. 14, 826.

[28] Q. Zhou, Q. Chen, Y. Tong, and J. Wang," Angew. Chem-Ger Edit,2016. 128, 11609.

[29] J. Barber and B. Andersson," Trends. Biochem. Sci.,1992. 17, 61.

[30] T. Ahmed, S. Balendhran, M. N. Karim, E. L. H. Mayes, M. R. Field, R. Ramanathan, M. Singh, V. Bansal, S. Sriram, M. Bhaskaran, and S. Walia," npj 2D Mater. Appl.,2017. 1, 18.

[31] A. Ziletti, A. Carvalho, D. K. Campbell, D. F. Coker, and A. C. Neto," Phys. Rev. Lett.,2015. 114, 046801.

[32] G. Wang, W. J. Slough, R. Pandey, and S. P. Karna," 2D Mater.,2016. 3, 025011.

[33] A. Carvalho and A. H. C. Neto, "Phosphorene: overcoming the oxidation barrier," vol. 1, ed: ACS. Cent. Sci, 2015, p. 289. 
[34] F. Alsaffar, S. Alodan, A. Alrasheed, A. Alhussain, N. Alrubaiq, A. Abbas, and M. R. Amer," Sci. Rep-UK,2017. 7.

[35] A. Favron, E. Gaufrès, F. Fossard, P. Lévesque, A. Phaneuf-L'Heureux, N. Tang, A. Loiseau, R. Leonelli, S. Francoeur, and R. Martel," arXiv preprint arXiv:1408.0345,2014.

[36] K. L. Utt, P. Rivero, M. Mehboudi, E. O. Harriss, M. F. Borunda, A. A. P. SanJuan, and S. Barraza-Lopez," 2015. 1, 320.

[37] Z. Hu, Q. Li, B. Lei, Q. Zhou, D. Xiang, Z. Lyu, F. Hu, J. Wang, Y. Ren, and R. Guo," Angew Chem Int Edit,2017. 56, 9131.

[38] S. Kim, J.-Y. Lee, C.-H. Lee, G.-H. Lee, and J. Kim," ACS Appl. Mater. Inter.,2017. 9, 21382.

[39] Y. Y. Illarionov, M. Waltl, G. Rzepa, T. Knobloch, J.-S. Kim, D. Akinwande, and T. Grasser," npj 2D Mater. Appl.,2017. 1, 23.

[40] X. Xiao, X. Liu, G. Cao, C. Zhang, L. Xia, W. Xu, and S. Xiao," Polymer Engineering \& Science, 2015. 55, 1296.

[41] H. Liang, A. Ueno, and K. Shinohara," Chem. Eng. Res. Des.,2000. 78, 49.

[42] A. Heuer," J. Eur. Ceram. Soc.,2008. 28, 1495.

[43] M. Zou, Y. Ma, X. Yuan, Y. Hu, J. Liu, and Z. Jin," J Semicond, 2018. 39.

[44] J. Ahmad, K. Bazaka, L. J. Anderson, R. D. White, and M. V. Jacob," Renew Sust Energ Rev,2013. 27.

[45] P. Gutruf, S. Walia, M. Nur Ali, S. Sriram, and M. Bhaskaran," Appl. Phys. Lett, 2014. 104.

[46] Y. Son, D. Kozawa, A. T. Liu, V. B. Koman, Q. H. Wang, and M. S. Strano," 2D Mater.,2017. 4, 025091.

[47] S. Gamage, A. Fali, N. Aghamiri, L. Yang, and P.-D. Ye," Nanotechnology,2017. 28, 26.

[48] V. Sorkin and Y. Zhang," Nanotechnology, 2017. 28, 075704.

[49] B. Wan, B. Yang, Y. Wang, J. Zhang, Z. Zeng, Z. Liu, and W. Wang," Nanotechnology, 2015. 26, 435702.

[50] J. Kim, S. K. Baek, K. S. Kim, Y. J. Chang, and E. Choi," Curr. Appl. Phys,,2016. 16, 165. 
[51] H. Zhu, S. McDonnell, X. Qin, A. Azcatl, L. Cheng, R. Addou, J. Kim, P. D. Ye, and R. M. Wallace," ACS Appl. Mater. Inter.,2015. 7, 13038.

[52] B. Wu, H. Zheng, Y. Ding, W. Liu, H. Lu, P. Zhou, L. Chen, Q. Sun, S. Ding, and D. W. Zhang," Nanoscale Res. Lett.,2017. 12, 282.

[53] Y. Cai, G. Zhang, and Y.-W. Zhang," J. Phys. Chem. C,2015. 119, 13929.

[54] X. Chen, Y. Wu, Z. Wu, Y. Han, S. Xu, L. Wang, W. Ye, T. Han, Y. He, and Y. Cai," Nat. Commun.,2015. 6, 7315.

[55] D. Yue, D. Lee, Y. D. Jang, M. S. Choi, H. J. Nam, D.-Y. Jung, and W. J. Yoo," Nanoscale, 2016. 8, 12773.

[56] D. Li, H. Jussila, L. Karvonen, G. Ye, H. Lipsanen, X. Chen, and Z. Sun," Sci. Rep-UK,2015. 5, 15899.

[57] C. Lin, R. Grassi, T. Low, and A. S. Helmy," Nano Lett., 2016. 16, 1683.

[58] D. Xiang, C. Han, J. Wu, S. Zhong, Y. Liu, J. Lin, X.-A. Zhang, W. P. Hu, B. Özyilmaz, and A. C. Neto," Nat. Commun.,2015. 6, 6485.

[59] Q. Li, Q. Zhou, X. Niu, Y. Zhao, Q. Chen, and J. Wang," J. Phys. Chem. Lett., 2016. 7, 4540.

[60] C. R. Ryder, J. D. Wood, S. A. Wells, Y. Yang, D. Jariwala, T. J. Marks, G. C. Schatz, and M. C. Hersam," Nat. Chem.,2016. 8, 597.

[61] V. Artel, Q. Guo, H. Cohen, R. Gasper, A. Ramasubramaniam, F. Xia, and D. Naveh," arXiv preprint arXiv:1611.03452,2016.

[62] S. Walia, S. Balendhran, T. Ahmed, M. Singh, C. El - Badawi, M. D. Brennan, P. Weerathunge, M. Karim, F. Rahman, A. Rassell, J. Duckworth, R. Ramanathan, G. E Collis, C. J Lobo, M. Toth, J. Christopher Kotsakidis, B. Weber, M. Fuhrer, J. M Dominguez - Vera, M. JS Spencer, I. Aharonovich, S. Sriram, M. Bhaskaran, and V. Bansal," Adv. Mater.,2017. 29, 1700152.

[63] W. Zhao, Z. Xue, J. Wang, J. Jiang, X. Zhao, and T. Mu," ACS Appl. Mater. Inter., 2015. 7, 27608 
[64] X. Tang, W. Liang, J. Zhao, Z. Li, M. Qiu, T. Fan, C. S. Luo, Y. Zhou, Y. Li, and Z. Guo," Small,2017. 1702739.

[65] F. Böhm, R. Edge, and T. G. Truscott," Acta. Biochm. Pol.,2012. 59, 27.

[66] M. B. Erande, M. S. Pawar, and D. J. Late," ACS Appl. Mater. Inter., 2016. 8, 11548.

[67] D. J. Late," Micropor. Mesopor. Mat.,2016. 225, 494.

[68] V. V. Chaban, E. E. Fileti, and O. V. Prezhdo," ACS Nano, 2017. 11, 6459.

[69] S. Nathiya, M. Durga, and T. Devasena," Analgesia,2014. 10.

[70] C.-A. Tseng, C.-C. Chen, R. K. Ulaganathan, C.-P. Lee, H.-C. Chiang, C.-F. Chang, and Y.-T. Chen," ACS Appl. Mater. Inter., 2017. 9.

[71] A. J. Mannix, X.-F. Zhou, B. Kiraly, J. D. Wood, D. Alducin, B. D. Myers, X. Liu, B. L. Fisher, U. Santiago, and J. R. Guest," Science,2015. 350, 1513.

[72] P. Vogt, P. De Padova, C. Quaresima, J. Avila, E. Frantzeskakis, M. C. Asensio, A. Resta, B. Ealet, and G. Le Lay," Phys. Rev. Lett.,2012. 108, 155501.

[73] M. Dávila, L. Xian, S. Cahangirov, A. Rubio, and G. Le Lay," New. J. Phys., 2014. 16, 095002.

[74] S. Balendhran, S. Walia, H. Nili, S. Sriram, and M. Bhaskaran," Small,2015. 11, 640.

[75] F.-f. Zhu, W.-j. Chen, Y. Xu, C.-I. Gao, D.-d. Guan, C.-h. Liu, D. Qian, S.-C. Zhang, and J.-f. Jia," Nat. Mater.,2015. 14, 1020.

[76] X.-L. Yu, L. Huang, and J. Wu," Physical Review B,2017. 95, 125113.

[77] C. Kamal and M. Ezawa," Physical Review B,2015. 91, 085423.

[78] S. Zhang, Z. Yan, Y. Li, Z. Chen, and H. Zeng," Angew. Chem-Ger Edit,2015. 127, 3155.

[79] G. Wang, R. Pandey, and S. P. Karna," ACS Appl. Mater. Inter.,2015. 7, 11490.

[80] E. Aktürk, O. Ü. Aktürk, and S. Ciraci," Physical Review B, 2016. 94, 014115.

[81] L. Lu, Z. Liang, L. Wu, Y. Chen, Y. Song, S. C. Dhanabalan, J. S. Ponraj, B. Dong, Y. Xiang, and F. Xing," Laser. Photonics. Rev, 2017.

[82] P. J. de Visser, R. Chua, J. O. Island, M. Finkel, A. J. Katan, H. Thierschmann, H. S. van der Zant, and T. M. Klapwijk," 2D Mater.,2016. 3, 021002. 
[83] N. Haratipour, M. C. Robbins, and S. J. Koester," arXiv preprint arXiv:1409.8395,2014.

[84] Y. Zhao, H. Wang, H. Huang, Q. Xiao, Y. Xu, Z. Guo, H. Xie, J. Shao, Z. Sun, and W. Han," Angew. Chem-Ger Edit,2016. 128, 5087.
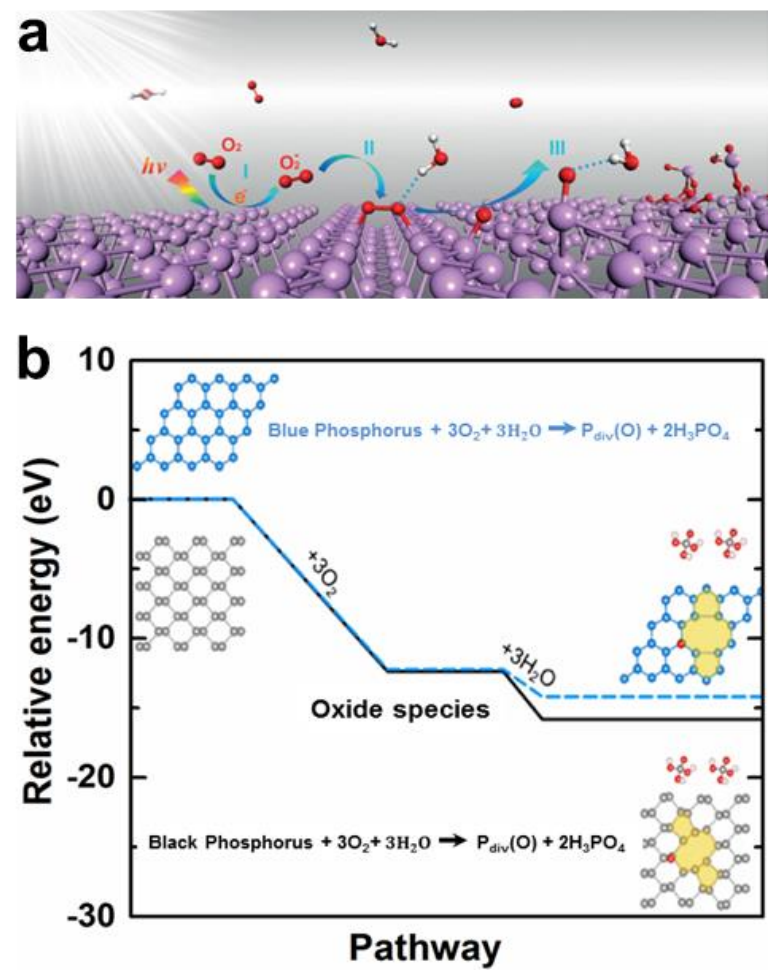

Figure 1: Schematic representations show: (a) a three-step process of BP degradation initiated by photo-oxidation. ${ }^{[28]}$ Copyright 2016, Wiley- Blackwell (b) influence of $\mathrm{O}_{2}$ and $\mathrm{H}_{2} \mathrm{O}$ on black and blue phosphorene with respect to relative energy. ${ }^{[32]}$ Image adapted from 2016, Institute of Physics Science Publishing. 


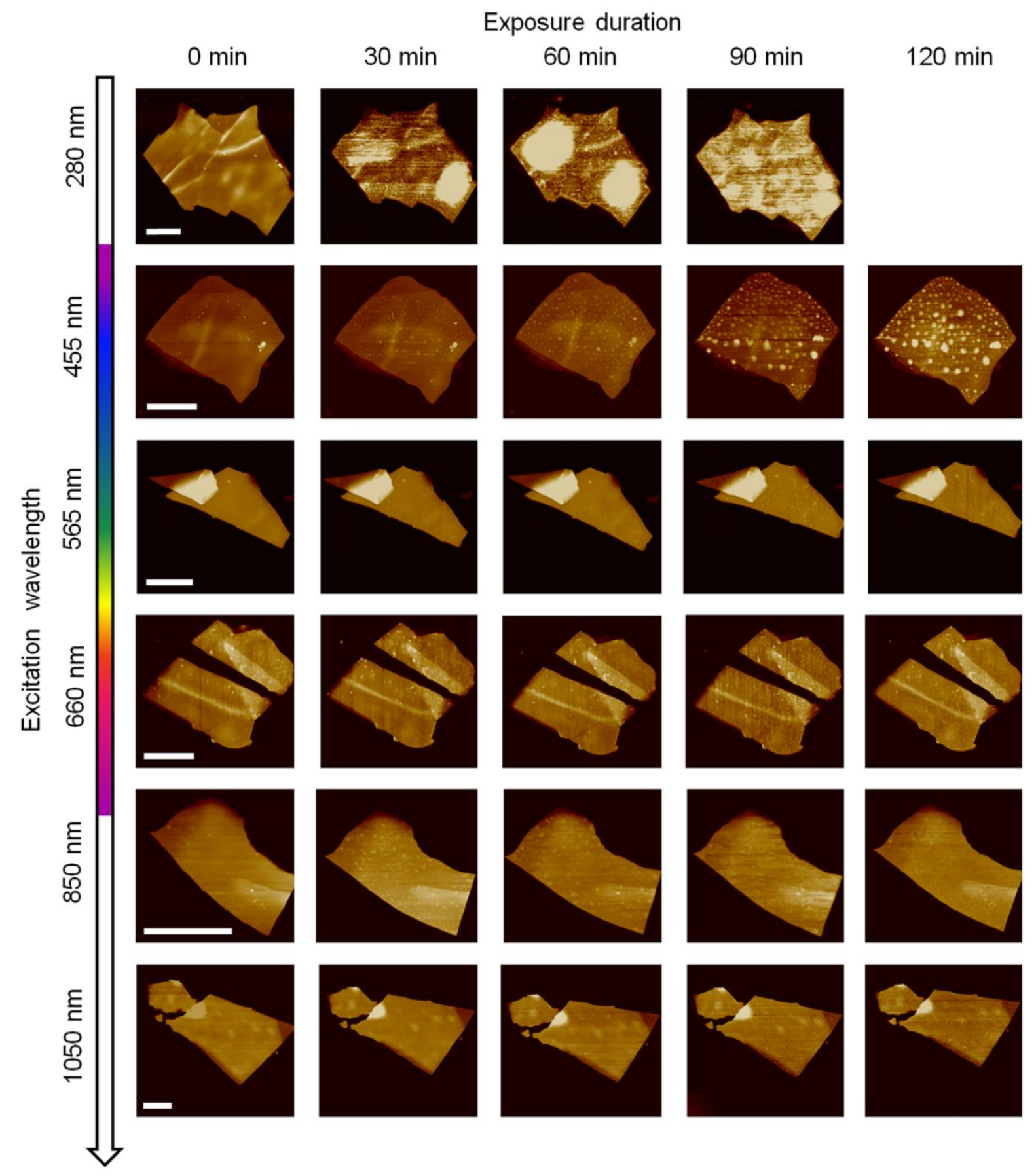

Figure 2: AFM images of BP upon exposure to various optical as a function of time. ${ }^{[30]}$ Copyright 2017, Nature Publishing Group. 

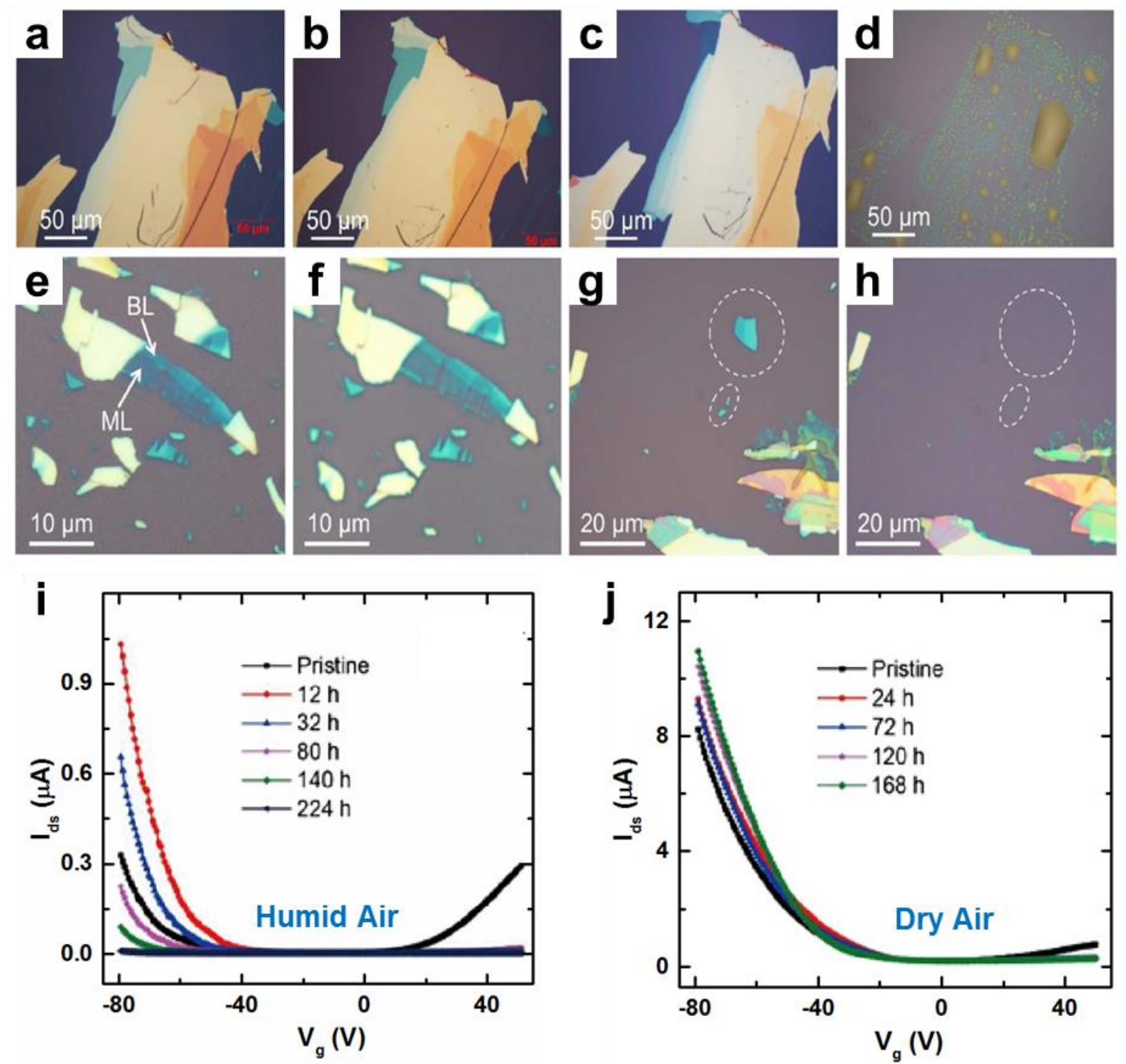

Figure 3: Optical images of (a) pristine BP flake, (b) the same flake after exposure to deionised water for 1 week, (c) same flake post-immersion in water for 2 weeks, (d) the same flake after exposure to air for 1 week. $^{[26]}$ (e) pristine BP flake, (f) same flake exposed to deaerated water for 2 days with $\mathrm{N}_{2}$ bubbling, (g) freshly exfoliated pristine BP flake followed by (h) immersion in oxygen rich water for 2 days. ${ }^{[26]}$ Copyright 2016, American Chemical Society. The transconductance of BP when exposed to (i) humid and (j) dry air in logarithmic and linear scale respectively at different control times with $V_{\mathrm{ds}}$ at $0.1 \mathrm{~V} .{ }^{[37]}$ Copyright 2017, Wiley-VCH. 

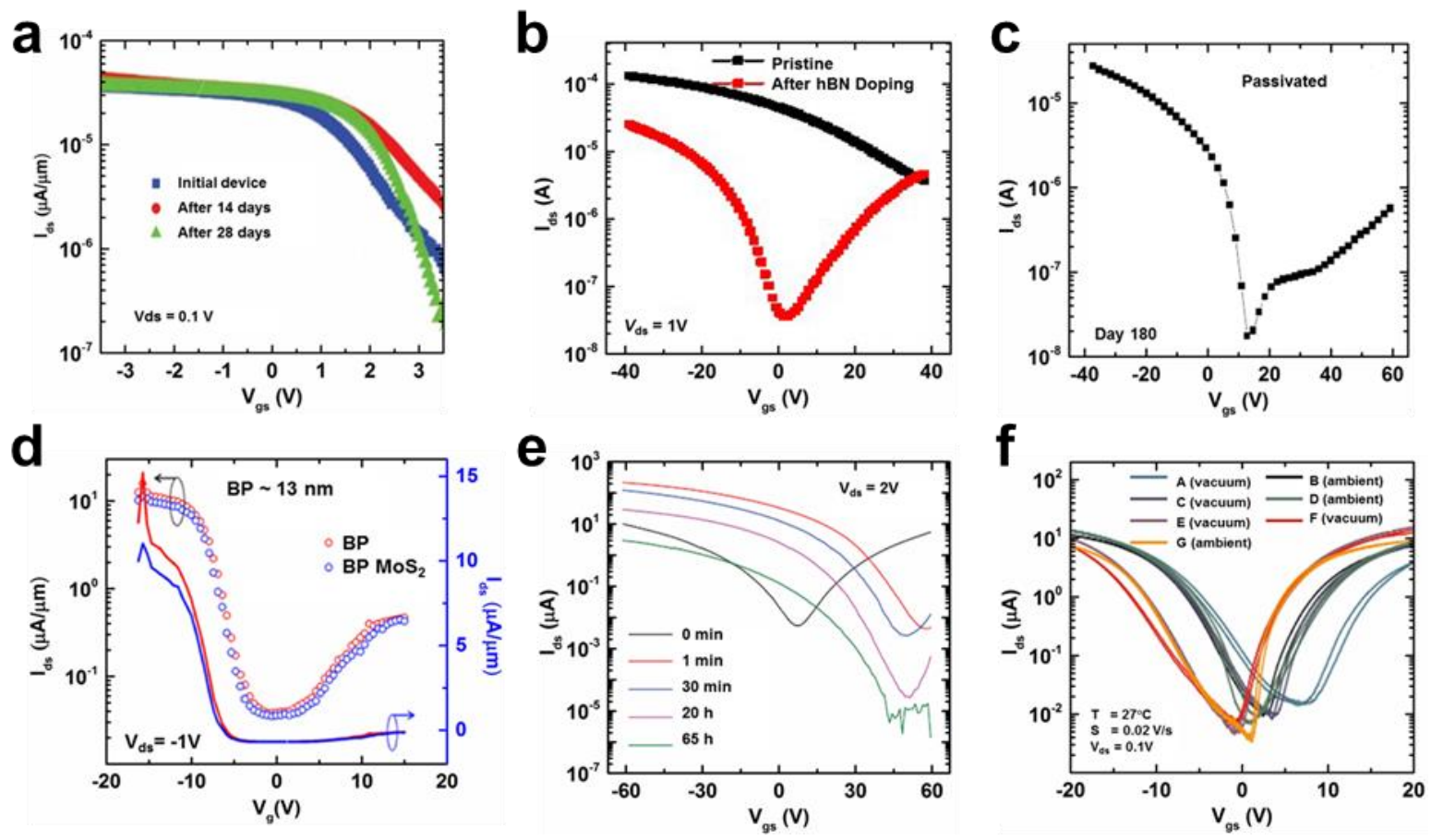

Figure 4: The transconductance curves of the (a) $\mathrm{BN} / \mathrm{Al}_{2} \mathrm{O}_{3}$ on BP FET devices at different time intervals, ${ }^{[47]}$ Image adapted from 2017 Institute Of Physics science Publishing. (b) The comparison of the pristine and h-BN capping layer on $\mathrm{BP},{ }^{[55]}$ (c) The BP layer passivated by benzyl viologen (BV) after 180 days, ${ }^{[55]}$ Copyright 2016, Royal Society of Chemistry (d) The comparison of pristine $\mathrm{BP}$ and $\mathrm{MoS}_{2}$ layer on $\mathrm{BP},{ }^{[46]}$ Image adapted from 2017, Institute of Physics Science Publishing (e) BP in 4-nitobenzene diazonium in different time frames, ${ }^{[60]}$ Copyright 2016, Nature Publishing Group (f) The $\mathrm{Al}_{2} \mathrm{O}_{3}$ coated BP in a long term study in different ambience showing up to 17 months preservation of BP. ${ }^{[39]}$ Copyright 2017 , Nature Publishing Group. 
a
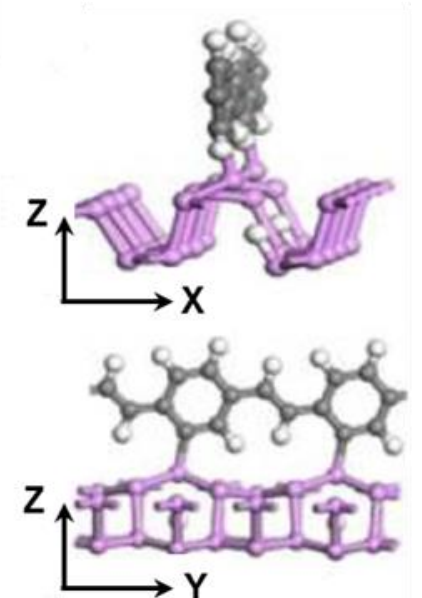

$\rightarrow$ Y

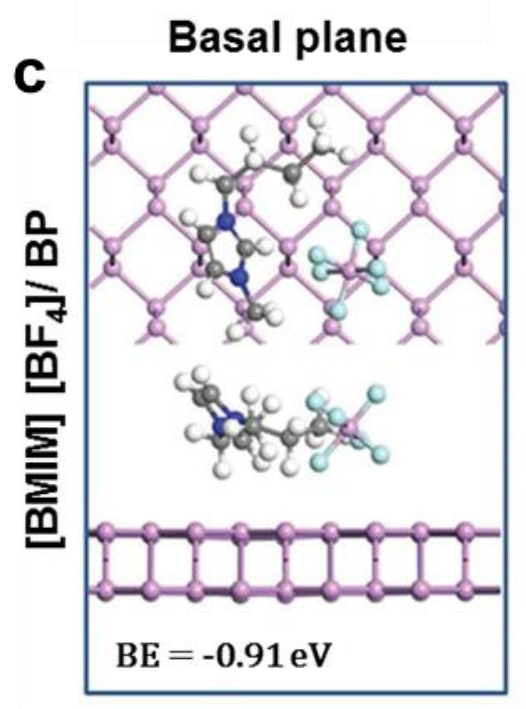

b

Pristine BP

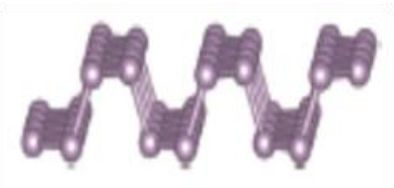

Edge plane

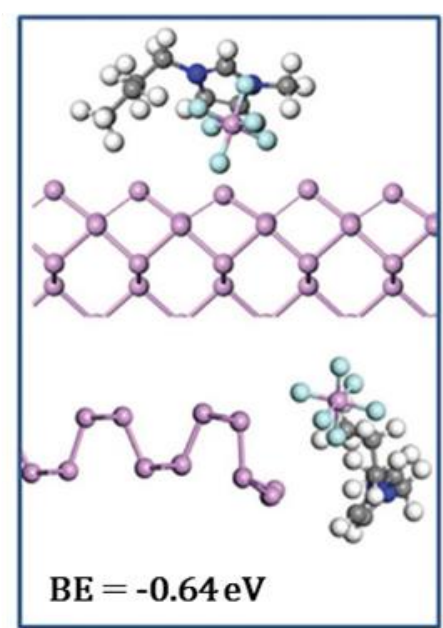

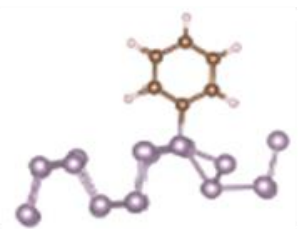

$\Delta E=-1.40 \mathrm{eV}$

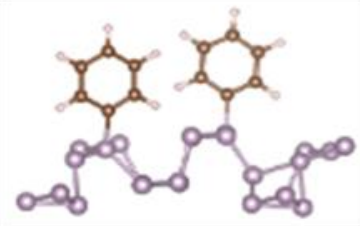

$\Delta E=-1.56 \mathrm{eV}$

Edge with two IL pairs

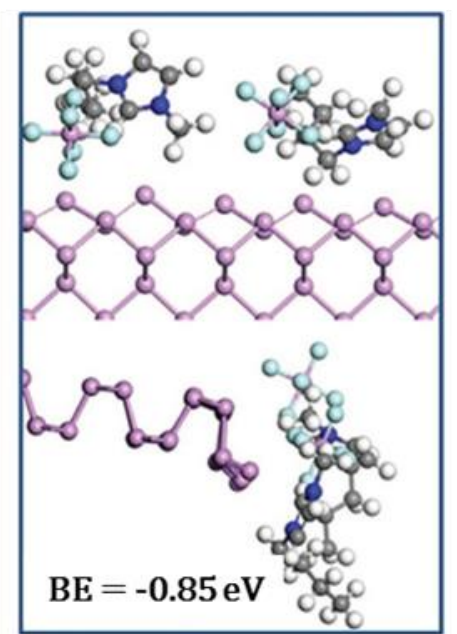

Figure 5: The DFT calculated structures of (a) Poly(phenylenevinylene) and BP along the zigzag direction viewing from the armchair direction on top and zigzag direction in the bottom ${ }^{[59]}$ Copyright 2016, American Chemical Society (b) The pristine BP (left) along the armchair view and covalent bonding of aryl groups to BP (right) ${ }^{[60]}$ Copyright 2016, Nature Publishing Group and (c) the structure of the strong chemisorption bonding of the [BMIM]$\left[\mathrm{BF}_{4}\right]^{[62]}$ on the BP basal plane, edge plane and the edge with two Ionic Liquids (IL) pairs, Copyright 2017, Wiley- Blackwell. 
Table 1: Protection strategies for BP reported so far with their experimented duration of stability and corresponding deposition and fabrication techniques.

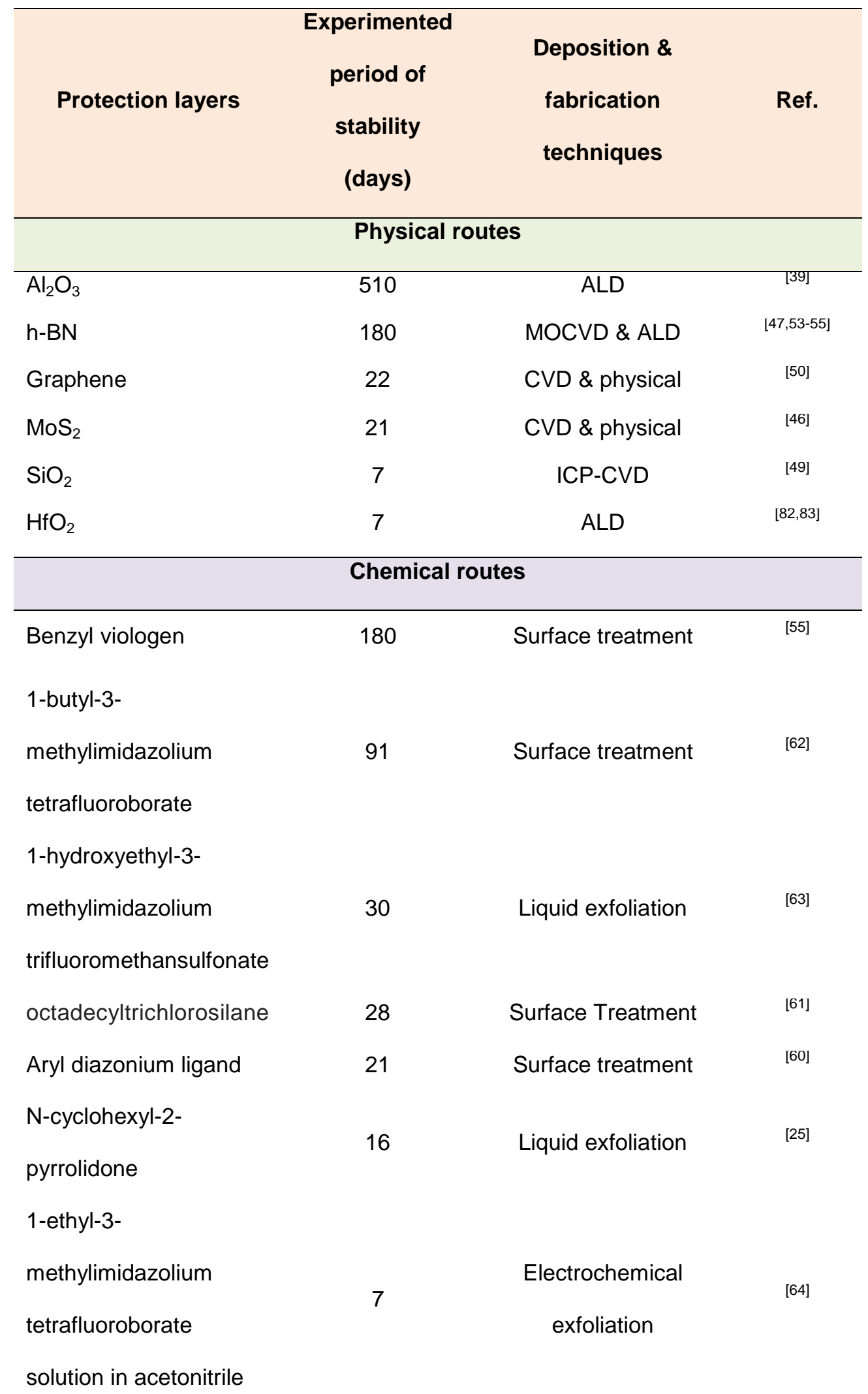


Titanium sulfonate 